\title{
Anabases
}

ANABASES Traditions et réceptions de l'Antiquité

$30 \mid 2019$

Varia

\section{Hélène en Égypte : Hérodote en dialogue avec l'épopée}

Arnaud Amilien

\section{OpenEdition}

1 Journals

Édition électronique

URL : https://journals.openedition.org/anabases/9772

DOI : 10.4000/anabases.9772

ISSN : 2256-9421

Éditeur

E.R.A.S.M.E.

Édition imprimée

Date de publication : 21 octobre 2019

Pagination : 53-67

ISSN : 1774-4296

\section{Référence électronique}

Arnaud Amilien, « Hélène en Égypte : Hérodote en dialogue avec l'épopée », Anabases [En ligne], 30

2019, mis en ligne le 21 octobre 2021, consulté le 08 novembre 2021. URL : http://

journals.openedition.org/anabases/9772 ; DOI : https://doi.org/10.4000/anabases.9772

(c) Anabases 

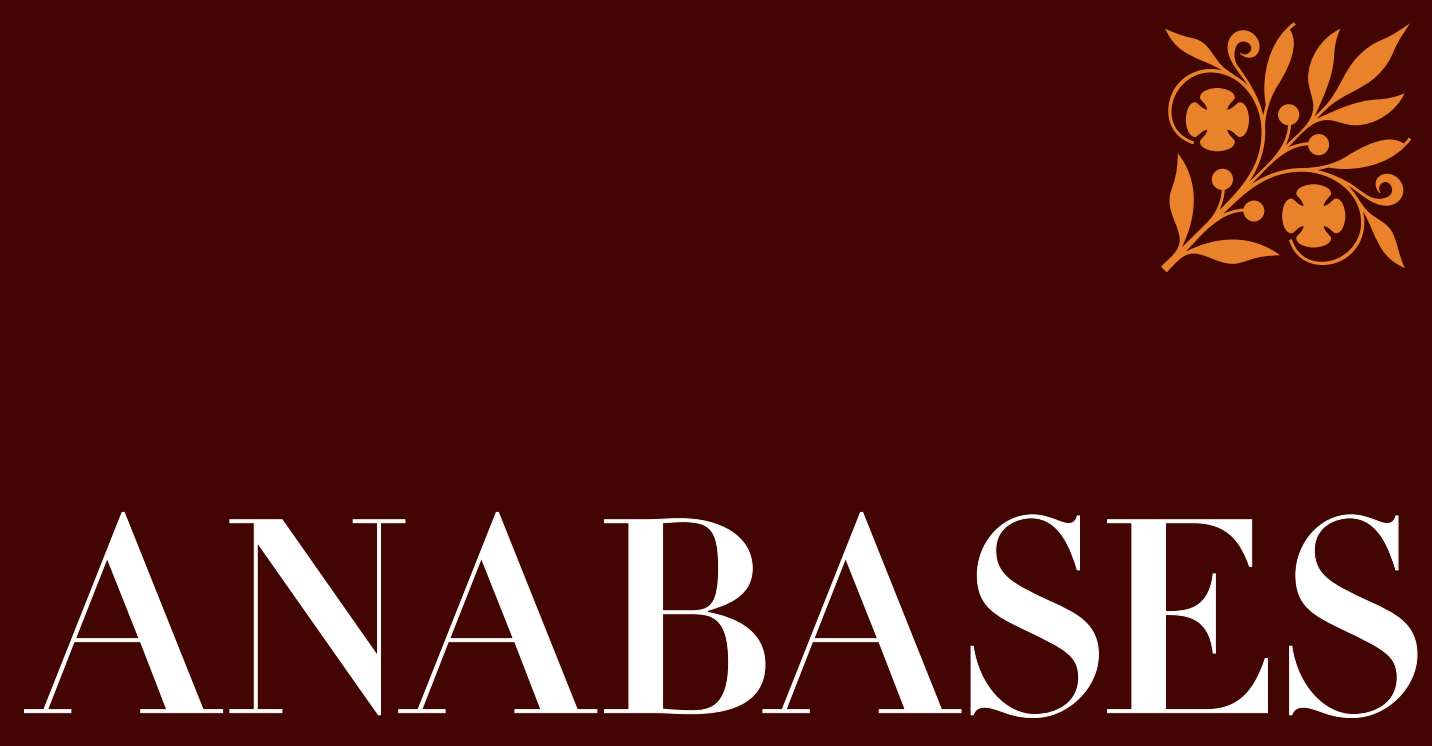

Traditions et Réceptions de l'Antiquité

\section{$\mathrm{N} \times 30$ \\ 2019}

Le sanctuaire de Gournay-sur-Aronde Le pindarisme et l'archéologie musicale Hélène en Égypte Perpétuer Ovide aux $X I V^{e}-X V I I I^{e}$ siècles II classico si fa pop Relire Marcel Detienne Freud à Pompéi 
ANABASES

Traditions et Réceptions de l'Antiquité

Revue de l'équipe de recherche E.R.A.S.M.E.

Université Toulouse-Jean Jaurès (UT2J)

Anabases dispose d'un Comité de lecture international. Chaque article envoyé à la rédaction est soumis, une fois anonymisé, à l'expertise de deux spécialistes qui rendent un rapport écrit. Les deux rapports anonymisés sont transmis à l'auteur qui tient compte des observations en vue de la publication.

\section{Comité SCIENTIFIQue}

Germaine Aujac (université Toulouse-Jean Jaurès : histoire de la géographie et des sciences antiques)

Florence Bouchet (université Toulouse-Jean Jaurès : littérature médiévale)

Hinnerk Brunns (CNRS : histoire économique et sociale ancienne et contemporaine)

Paulo Butti de Lima (université de Bari : historiographie et réception de l'Antiquité)

Luciano CANFora (université de Bari : littérature et histoire anciennes, historiographie)

Giovanna Ceserani (Stanford University : histoire intellectuelle et historiographie de la tradition classique)

Temístocles Cezar (université de Porto Alegre : historiographie moderne)

Serafina Сuомо (University of London, Birkbeck College : histoire des mathématiques et des sciences)

Paul Demont (université de Paris Sorbonne : philologie grecque et héritage classique)

Marie-Laurence Desclos (université de Grenoble II : philosophie de l'Antiquité)

Olivier Devillers (université de Bordeaux 3 - Michel-de-Montaigne : littérature et historiographie latines)

Andrea Giardina (Istituto italiano di scienze umane : histoire du monde romain et de ses réceptions)

Ève Gran-Aymerich (AIBL : histoire de l'archéologie et des transferts culturels)

François Hartog (eHess : historiographie ancienne et moderne)

Geneviève Hoffmann (université de Picardie : histoire des mondes grecs)

Christian JACOB (CNRS/EHESS : histoire comparée et épistémologie des savoirs)

Suzanne Marchand (Louisiana State University : histoire du classicisme et de l'orientalisme)

Wilfried NIPPEL (Humboldt Universität Berlin : histoire et historiographie de l'Antiquité)

Sylvie Pitria (université de Paris I-Panthéon Sorbonne : histoire et historiographie du monde romain)

Stéphane Ratтi (université de Franche-Comté - Besançon : philologie et héritage latin)

Comité de RÉdACtion

Clément Bertau-Courbières, Corinne Bonnet, Laurent bricault, Clément Bur, Adeline Grand-Clément, Anne-Hélène Klinger-Dollé, Véronique Krings, Thibaud Lanfranchi, Pascal Payen, Grégory Reimond, Sarah Rey, Catherine Valenti, Noémie VillacèQue

ÉditeUr RESPONSABLE

Clément Bur

Éditrice ADJOINTE

Catherine VALENTI

Sites Web

http://plh.univ-tlse2.fr

Revues.org : http://anabases.revues.org

Aвonnement et vente aU numéro

Éditions De Boccard - 4, rue de Lanneau - 75005 Paris

info@deboccard.com - www.deboccard.com

Tél. : 0033/(0)143260037 - Fax : 0033/(0)143548583 




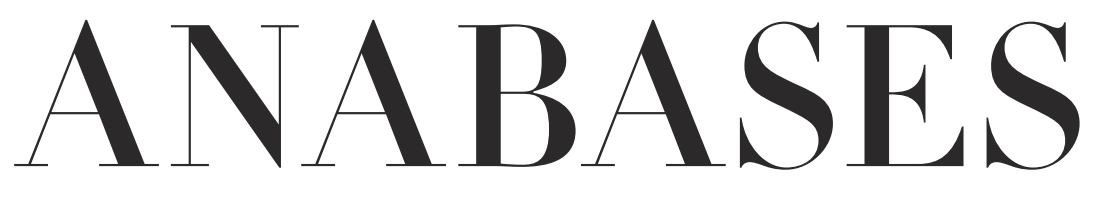

Traditions et Réceptions de l'Antiquité

$$
\begin{aligned}
& N \circ 30 \\
& 2019
\end{aligned}
$$

\section{E.R.A.S.M.E.}

Université Toulouse - Jean Jaurès 



\section{Sommaire}

ํo30 - 2019

\section{Historiographie et identités culturelles}

\section{Carole Quatrelivre}

Le sanctuaire gaulois de Gournay-sur-Aronde (Oise).

Retour sur une découverte exceptionnelle des années I970 . . . . . . . . II

\section{Traditions du patrimoine antique}

Christophe Corbier

Le pindarisme et l'archéologie musicale : style, valeur et authenticité

de la première Pythique à l'époque moderne . . . . . . . . . . . . . . .

Arnaud AmiLIEN

Hélène en Égypte : Hérodote en dialogue avec l’épopée . . . . . . . . . .

\section{Archéologie des savoirs}

Sébastien Cazalas

Au jardin des exempla. Rhétorique et stratégie de l'exemplum antique

dans l'œuvre politique de Jean Juvénal des Ursins (I388-ı473) . . . . . . 7 7

Dossier - Perpétuer Ovide : aspects moraux, éditoriaux,

linguistiques et culturels $\left(\mathrm{XIV}^{\mathrm{e}}-\mathrm{XVIII}^{\mathrm{e}} \mathrm{s}\right.$.)

Francesca Dell'Oro

Introduction $\ldots \ldots \ldots \ldots$. . . . . . . . . . . . . . 89

Hélène Casanova-Robin

L’audace châtiée : Phaéton, Actéon et Icare dans la tradition latine jusqu'à la Renaissance, tours et détours d'un symbolisme . . . . . . . . 9 93 
Dylan Bovet

Le commentaire latin des Métamorphoses d'Ovide : pratiques humanistes et évolutions de Regius-Micyllus (I543) à Burmann-Heinsius (I727) . . . . III

Martine FurNo

Ovide en classe, ou un auteur en éclats . . . . . . . . . . . . . I27

Basil NELIS

D’un Ovide chrétien à un Ovide burlesque, du Moyen Âge au Grand Siècle : continuités et changements dans la traduction et dans l'illustration des Métamorphoses perçus à travers deux éditions du xvII ${ }^{\mathrm{e}}$ siècle . . . . . . . I I43

Olivier ThÉvenAz

Épilogue ....................... I6

\section{Actualités et débats}

Tiphaine-Annabelle BEsnard

2019 : l'année pop des musées d'archéologie. Retour sur l'exposition

romaine Il classico sifa pop. Di scavi, copie e altripasticci . . . . . . . . . . I I7I

\section{Lire, relire la bibliothèque des sciences de l'Antiquité}

Jean-Pierre Albert

Le premier Detienne : une relecture de

“ La notion mythique d"A $\lambda \hat{n} \theta \varepsilon 1 \alpha$ » $\left(R E G\right.$, I96o, p. 27-35) . . . . . . . . . . $\quad{ }_{779}$

Marcel Detienne

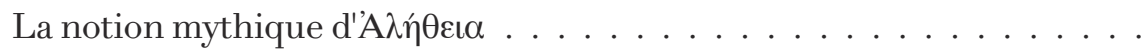

\section{Ateliers de l'histoire}

Antiquités numériques (coordonné par Elodie Guillon) ( $n^{\circ} 1$ )

Élodie GuILlon

Introduction

Jaime Alvar

Le projet EPIDI : Epítetos divinos.

Experiencia religiosa y relaciones de poder en Hispania . . . . . . . . . . . I98 Les mots de l'Antiquité (coordonné par Magali Soulatges) ( $n^{\circ} 10$ )

Jack Thомаs

L’Antiquité dans les toponymes de l'État de New York . . . . . . . . . . . 202

Actualité du théâtre (coordonné par Malika Bastin-Hammou) ( $\left.n^{\circ} 4\right)$

Mathieu FERrand

“ Avons-nous perdu le Soleil ? / Ou l'avons-nous chassé ? » 
Thyeste de Sénèque, traduit par Florence Dupont.

Mise en scène de Thomas Jolly (Avignon, 20I8) . . . . . . . . . . . . . 2 2I4

Voyages et Voyageurs (coordonné par Véronique Krings) ( $n^{\circ} 11$ )

Claude AzIzA

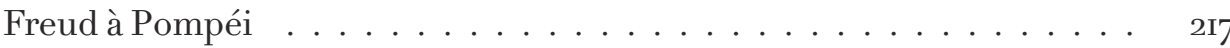

\section{Comptes rendus}

Lucile Arnoux-Farnoux et Polina Kosmadaki (dir.)

Le double voyage : Paris-Athènes (Igrg-Ig39) (Catherine Valenti) . . . . . . 225

Sandra Boehringer et Daniele Lorenzini (dir.)

Foucault, la sexualité, l'Antiquité (Jan Nelis) . . . . . . . . . . . . . . . . 226

Corinne Bonnet, Nicole Belayche, Marlène Albert Llorca,

Alexis Avdeeff, Francesco Massa, Iwo Slobodzianex (dir.)

Puissances divines à l'épreuve du comparatisme. Constructions,

variations et réseaux relationnels (Geneviève Hoffmann) . . . . . . . . . . 228

Shane ButLer (éd.)

Deep Classics, Rethinking Classical Reception (Jan Nelis) . . . . . . . . . . 23o

Zeynep ÇELIK

About Antiquities: Politics of Archaeology in the Ottoman Empire (Jorge Elices Ocón) . . . . . . . . . . . . . . . . . 231

Xavier Deru et Germaine Leman-Delerive (éd),

Franz Cumont, Comment la Belgique fut romanisée (Vivien Barrière) . . . 234

Olivier Devillers, Breno Battistin Sebastiani (éd.)

Sources et modèles des historiens anciens (Arnaud Saura-Ziegelmeyer) . . $\quad 235$

Mara Fazio, Pierre Frantz et Vincenzo De Santis (dir.)

Les Arts du spectacle et la référence antique dans le théâtre

européen (I760-I830) (Arnaud Saura-Ziegelmeyer) . . . . . . . . . . . . . . 237

Jérémy Guedu et Barbara Meazzi (dir.)

La culture fasciste entre latinité et méditerranéité (I880-1940), in Cahiers de la Méditerranée 95, (Andrea Avalli) . . . . . . . . . . . . . . . 239

Marie-Laurence HAAck (éd.), avec la collaboration de Martin MiLler, Les Étrusques au temps du fascisme et du nazisme (Jan Nelis) . . . . . . . . . 24I

Arlene Holmes-Henderson, Steven Hunt et Mai Musié (éd.)

Forward with Classics. Classical Languages in Schools and Communities

(Charlotte Tournier) . . . . . . . . . . . . . . . . . . . . . . .

Daniel Jew, Robin Osborne et Michael Scotт (éd.)

M. I. Finley. An Ancient Historian and his Impact (Hinnerk Bruhns) . . . . $\quad 244$ 
Kostas Kalimtzis,

An inquiry into the philosophical concept of scholê.

Leisure as a Political End (Florent Rouzade) . . . . . . . . . . . . . . . . . 246

Anne-Hélène KLINGER-Dollé

Le De sensu de Charles de Bovelles. Conception philosophique

des sens et figuration de la pensée. Suivi du texte latin du De sensu,

traduit et annoté (Laure Hermand-Schebat) . . . . . . . . . . . . . 248

Egidia Occhipinti

The Hellenica Oxyrhynchia and Historiography:

New Research Perspectives (Anne de Cremoux) . . . . . . . . . . . . . . . . 249

Laurent OLIVIER (dir.)

La mémoire et le temps. L'ouvre transdisciplinaire

d'Henri Hubert (I872-I927) (Sarah Rey) . . . . . . . . . . . . . . . 25I

Gabriella Pinonti et Corinne Bonnet (dir.),

Les dieux d'Homère. Polythéisme et poésie en Grèce ancienne,

Kernos, Supplément 3r. (Sandya Sistac) . . . . . . . . . . . . . . . 253

Rabun TAYLoR, Katherine W. Rinne et S. Kostof

Rome. An Urban History from Antiquity to the Present

(Cyrielle Landrea) . . . . . . . . . . . . . . . . . . . 255

Wyger Velema et Arthur Weststeisn (éd.)

Ancient Models in the Early Modern Republican Imagination

(Paulo Butti de Lima) . . . . . . . . . . . . . . . . . . . 256

Philip WaLsh (éd.)

Brill's Companion to the Reception of Aristophanes

(Malika Bastin-Hammou) . . . . . . . . . . . . . . .

Richard WARren

Art Nouveau and the Classical Tradition (Lucien Calvié) . . . . . . . . . 26o

Jesse Weiner, Benjamin Eldon Stevens et Brett M. Rogers (éd.)

Frankenstein and Its Classics. The Modern Prometheus

from Antiquity to Science Fiction (Mathieu Scapin) . . . . . . . . . . . . . 26r

Nigel G. WiLson

From Byzantium to Italy. Greek Studies in the Italian Renaissance,

deuxième édition (Luigi-Alberto Sanchi) . . . . . . . . . . . . . . 262

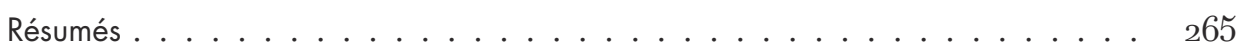

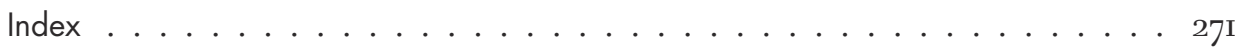




\section{Traditions}

du patrimoine antique 

Anabases 30 (2019), p. 53-67.

\section{Hélène en Égypte : Hérodote en dialogue avec l'épopée}

Arnaud Amilien

I e célèbre passage de l'Enquête relatif à l'aventure d'Hélène et Ménélas en Égypte (II, 112-120) est considéré comme un exemple emblématique de la méthode historique d'Hérodote. De nombreux philologues ont en effet voulu y reconnaitre une démarche de recherche motivée par la rationalité, qui se matérialisait par une prise de distance critique face au mythe et une remise en question des poètes épiques ${ }^{1}$. Ainsi, face au témoignage des prêtres égyptiens, lui livrant une variante de l'histoire d'Hélène fort différente de celles qu'il connaissait ${ }^{2}$,

1 Ainsi en est-il de H. VERDin, « Les remarques critiques d'Hérodote et Thucydide sur la poésie en tant que source historique », Historiographia antiqua: commentationes Lovanienses in honorem W. Peremans septuagenarii editae, Leuven, Leuven University Press, coll. "Symbolae Facultatis Litterarum et Philosophiae Lovaniensis », 1977, p. 60-61 ; C. Brillante, “ Myth and History: History and the Historical Interpretation of Myth », in L. Edmunds (éd.), Approaches to Greek Myth, Baltimore, JHU Press, 1990, p. 91-140 ; D. Boedecker, “Herodotus' Genre(s) », in M. Depew - D. Obbink (éds), Matrices of Genre. Authors, Canons, and Society, Cambridge (MA), Harvard University Press, 2000, p. 103-105; D. Boedecker, “Epic Heritage and Mythical Patterns in Herodotus ", in E. J. Bakker - I. J. F. de Jong - H. van Wees (éds), Brill's Companion to Herodotus, Leyde - Boston - Cologne, Brill, 2002, p. 108.

2 Il existait en effet différentes versions de cette histoire dans plusieurs genres littéraires. Outre Homère (Od., IV, 227-228), on sait que Stésichore d'Himère (Page, $P M G$, F 15), Hécatée de Milet (FGrH 1 F 307-308) et Hellanicos de Lesbos (FGrH 4 F $153)$ ont mentionné le passage d'Hélène sur la terre égyptienne, même si le caractère fragmentaire des sources rend difficile de savoir ce que chacun avait exactement raconté à ce propos. Pour une liste complète des testimonia portant sur ce récit, voir la notice de Ph.-E. Legrand dans son édition du livre II d'Hérodote (Hérodote. Histoires, 
l'enquêteur en vient à leur demander ce qu'ils pensent de la guerre de Troie (II, $117,1)$, laissant entendre que le récit des Grecs pourrait être insensé ( $\mu$ ó $\tau \alpha \iota v){ }^{3}$, ce qui en dit déjà long sur sa posture. Homère - entendons par là le poète qui, selon les Anciens, a composé les deux épopées homériques ${ }^{4}$-, qui avait longtemps été considéré comme le socle même du savoir dans le monde grec, semble donc avoir perdu son statut de “ maître de vérité » ${ }^{5}$, étant apparemment négligé face au clergé égyptien. Certains chercheurs ont par ailleurs suggéré que cette méthode critique, consistant à hiérarchiser ses sources et à accorder la primauté aux plus fiables, pouvait faire figure d'argument d'autorité, mettant en exergue la rigueur dont a fait preuve l'auteur durant ses investigations ${ }^{6}$. Dès lors, la tentation est

II, Paris, Les Belles Lettres, CUF, 1932, p. 32-33) et le commentaire de A. B. Luoyd (Herodotus. Book II, Commentary 99-182, Leyde - New York - Copenhague - Cologne, Brill, 1988, p. 46-47). D. Fehling (Herodotus and his « Sources »: Citation, Invention, and Narrative Art, Leeds, Cairns, 1989, p. 59-60), lui, aborde ces témoignages avec un regard critique dans l'optique d'évaluer leur influence sur le “ père de l'histoire ».

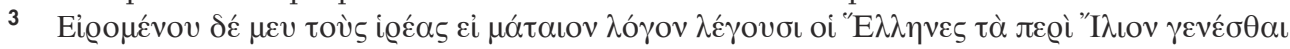
ทे oủ (“Tandis que j'interrogeais les prêtres pour savoir si les Grecs tenaient ou non un discours insensé à propos de ce qui s'est passé à Troie »).

4 Il n'est nul besoin, selon nous, de revenir ici sur la question homérique qui n'a aucun lien avec notre propos. Si nous voulons étudier la manière dont Hérodote “ dialogue » avec l'épopée, il faut garder à l'esprit que l'historien grec considérait les deux épopées homériques comme des pièces dues à un seul et même poète, ignorant la théorie moderne selon laquelle elles se seraient toutes deux constituées par strates successives. Pour un état détaillé de la question homérique, nous renverrons le lecteur à A. Ballabriga, "La question homérique : pour une réouverture du débat », REG 103 (1990), p. 16-29 ; G. NAGY, Homeric Questions, Austin, University of Texas Press, 1996 ; S. SAÏD - M. TrédÉ - A. Le Boulluec, Histoire de la littérature grecque, Paris, PUF, coll. " Quadrige manuels », 1997, p. 13-24; R. Fowlen, “ The Homeric Question », in R. Fowler (éd.), The Cambridge Companion to Homer, Cambridge, Cambridge University Press, 2004, p. 220-232 ; L. Ferreri, La questione omerica dal Cinquecento al Settecento, Roma, Edizioni di storia e letteratura, 2007.

5 Cette expression reprend sciemment le titre d'un ouvrage de M. DEtiEnne (Les maîtres de vérité dans la Grèce archaïque, Paris, Maspero, 1967), dont une importante partie est consacrée au statut du rhapsode dans la société mycénienne.

6 Cette position a été adoptée par M. I. Finley, "Myth, Memory, and History », in M. I. Finley (éd.), The Use and Abuse of History, New York, The Viking Press, 1975, p. 14 ; K. H. Waters, Herodotus the Historian: His Problems, Methods and Originality, Londres - Sydney, Routledge, 1985, p. 76 ; D. Lateiner, The Historical Method of Herodotus, Toronto, University of Toronto Press, 1989, p. 63 ; R. Thомas, “ Performance and Written Publication in Herodotus and the Sophistic Generation ", in W. Kullmann - J. Althoff (éds), Vermittlung und Tradierung von Wissen in der griechischen Kultur, 
grande de voir dans cette attitude l'un des avatars du passage du $\mu \hat{v} \theta$ o pour reprendre le titre de l'ouvrage de Wilhelm Nestle ${ }^{7}$.

Cependant, il faut rappeler que la prudence reste de mise face à de telles interprétations, puisque cette prétendue «transition » d'une forme de pensée à l'autre a donné lieu à des objections. Affirmera-t-on que le discours mythique est dépourvu de rationalité ${ }^{8}$ ? Et osera-t-on dire que le mythe n'a plus sa place dans la société contemporaine d'Hérodote 9 ? Si cette vision a longtemps prévalu, on a pu contester cette conception binaire d'une rupture entre la légende et le discours raisonné, entre le rhapsode des temps anciens et l'historien de l'époque classique $^{10}$. Il ne faudrait pas, en effet, perdre de vue que nous nous trouvons, dans l'Enquête, en présence d'un processus lent toujours en cours. Si Hérodote tente de faire dialoguer ses sources dans sa recherche de la vérité historique, il le fait avec sa propre forme de rationalité (ou plutôt celle de son époque ?) qui n'est clairement pas la nôtre. Cela se manifeste, entre autres, dans le rapport entretenu avec la tradition aédique. Loin d'avoir renié le mythe, la période classique, que l'on perçoit souvent comme un premier « mouvement des Lumières »", témoigne

Script-Oralia 61, Tübingen, 1993, p. 241; J. Marincola, Authority and Tradition in Ancient Historiography, Cambridge, Cambridge University Press, 1997, p. 100 ; P. Cartledge - E. Greenwood, “ Herodotus as a Critic: Truth, Fiction, Polarity », in E. J. Bakker - I. J. F. de Jong - H. van Wees (éds), Brill's Companion to Herodotus, Leyde Boston - Cologne, Brill, 2002, p. 355.

7 W. Nestle, Vom Mythos zum Logos, Stuttgart, Kröner, 1940.

8 Nous ne reviendrons pas ici sur cette question, déjà traitée par L. Couloubaritsis, Aux origines de la pensée européenne, de la pensée archaïque au néoplatonisme, Bruxelles, De Boeck-Wesmael, 20034, p. 57-70. Selon ce philosophe, il y aurait dans le mythe une autre forme de logique, qualifiée de “logique de l'ambivalence », ce qui interdit de penser que la pensée rationnelle est apparue ex nihilo.

9 L'excellente synthèse de BoEDEcker, « Epic Heritage », portant sur l'héritage mythique dans l'Enquête, pousse à croire le contraire.

10 Dans l'introduction de son livre Mythologies of Transhumanism, intitulée “From Logos to Mythos ", M. Hauskeller (Bâle, Springer International Publishing AG, 2016, p. 1-10) répond à l'ouvrage de Nestle, Vom Mythos, affirmant que le passage du mythe à la pensée rationnelle, de la subjectivité à l'objectivité, n'est pas et ne peut jamais être total.

1 Dans un ouvrage intitulé Les Origines de la pensée grecque, J.-P. Vernant (Paris, PUF, coll. " Mythes et Religions », 1962) essaie de décrire le passage de la pensée mythique aux savoir rationnels, en se demandant quelles conditions sociales et historiques ont pu provoquer cette évolution. Cet avènement de la « rationalité » a été par la suite relativisé, puisque celle-ci ne naît pas toute formée de nulle part : les Grecs ont développé leur " science » au contact des savoirs des sociétés égyptienne et babylonienne, même si la pensée hellénique se distingue par une focalisation sur la recherche des causes qui 
envers lui d'une attitude équivoque. S'ils s'en détachent sur certains plans, les auteurs continuent à puiser leur inspiration dans l'héritage mythique et à le réinterpréter, l'employant comme une structure de base sur laquelle viennent se greffer de nouvelles réflexions. Platon, dans la République, fournit un exemple fort révélateur de ce phénomène, lui qui, tout en dénonçant les conceptions des dieux néfastes à la pureté de l'âme, reconnaît - certes, sur un ton légèrement ironique qu'il y a beaucoup à louer chez Homère ${ }^{12}$. Son admiration pour le poète apparaît d'ailleurs plus manifestement encore dans l'Ion, où Socrate l'appelle « le meilleur et le plus divin des poètes ${ }^{13}{ }^{13}$. L'ironie sous-jacente n'enlève rien à la place de choix que le philosophe accorde au rhapsode.

C'est dans cette mouvance qu'il convient de situer l'Enquête. Bien sûr, il semble difficile de contester qu'Hérodote mette explicitement en avant le caractère douteux des récits épiques, comme nous l'avons vu dans l'extrait mentionné plus haut. Cela n'empêche pour autant pas qu'il reconnaisse aussi à Homère une certaine légitimité scientifique, comme l'atteste le passage relatif à la poussée des cornes des bœufs, où le témoignage du poète est cité pour justifier son affirmation $(\mathrm{IV}, 29)^{14}$. À une époque où la pensée que nous avons pris l'habitude de qualifier de “ scientifique » a émergé, il est notable de trouver une telle référence à la poésie épique pour expliquer un phénomène physique. Ce constat donne à penser que l'œuvre d'Homère, quoique remise en question, n'en était pas moins considérée comme une source, ne fût-ce que partiellement. Cette posture assez ambivalente

ne semble pas avoir d'équivalent dans le monde oriental (J.-F. Dortier, “Y a-t-il eu un miracle grec? ", in T. LePeltien (éd.), Histoire et philosophie des sciences, Auxerre, Sciences Humaines Éditions, 2013, p. 11-20).

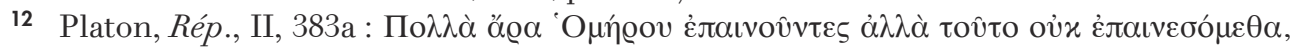

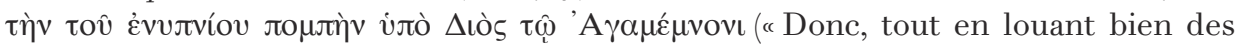
choses chez Homère, nous n'approuverons pas cela, je veux dire le fait que Zeus ait envoyé le songe à Agamemnon »).

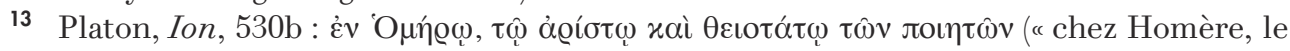
meilleur et le plus divin des poètes »).

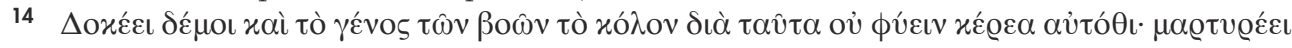

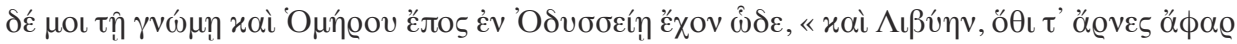

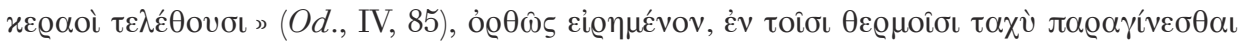

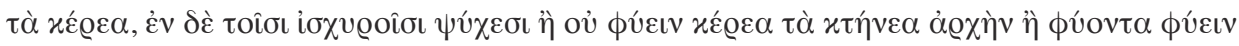

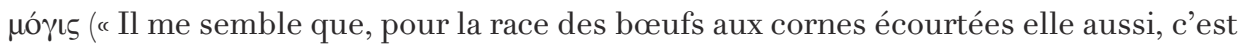
pour cette même raison que les cornes ne poussent pas. Pour moi, cette opinion est également confirmée par le vers homérique tiré de l'Odyssée que voici : 'et la Libye, où les agneaux sont immédiatement pourvus de cornes'. Il est bien dit que, dans les lieux chauds, les cornes apparaissent rapidement tandis que, dans les zones rigoureusement froides, soit elles ne poussent pas du tout au bétail soit, si elles poussent, ce n'est qu'à peine »). 
remonte apparemment à Hésiode, qui présente les Muses comme capables de mentir quand elles le souhaitent ${ }^{15}$. Cette image signifierait que les Grecs savaient que les récits des aèdes comprenaient des éléments trompeurs, tout en croyant qu'un fond de vérité se dissimulait sous le voile de la faussetél6.

Hérodote ne semble pas faire exception à la règle, comme le montrent les chapitres 112 à 120 du livre II de l'Enquête. Si l'historien n'hésite pas à y contester Homère, en affirmant que l'épouse de Ménélas n'est jamais allée à Troie (II, 120), il semble cependant manifester, comme nous allons le voir, une volonté de faire coïncider le témoignage du rhapsode avec celui des prêtres égyptiens. Comment comprendre une telle attitude, teintée d'ambiguïté ? L'auteur apporte peut-être lui-même une réponse à cette question :

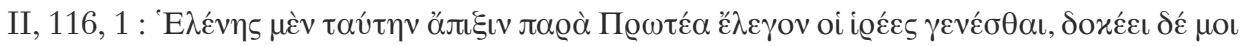

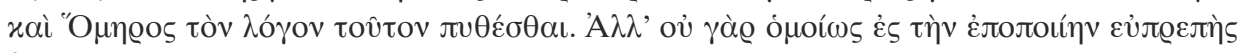

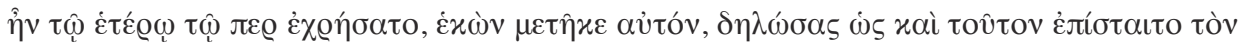

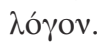

“ Les prêtres disaient qu'Hélène était arrivée ainsi auprès de Protée, mais il me semble qu'Homère lui aussi avait pris connaissance de cette histoire. Mais, comme elle ne convenait pas aussi bien à l'épopée que l'autre histoire qu'il a effectivement développée, il l'a volontairement laissée de côté, non sans avoir montré qu'il connaissait aussi cette histoire. » (Traduction personnelle)

Dans ce passage, il est à noter qu'Hérodote semble pleinement conscient de l'existence d'un genre épique, un genre qu'il ne reconnaît pas comme le sien. Cela apparaît clairement un peu plus loin, où il se demande « s'il faut avancer quelque

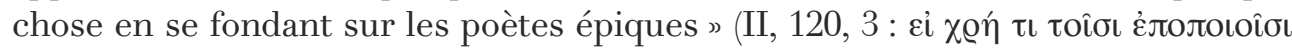

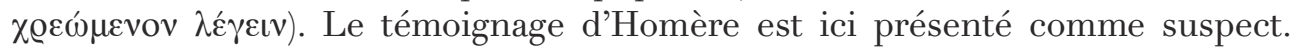
Pourtant, l'historien reconnaît que ce poète avait eu vent du passage d'Hélène dans les contrées égyptiennes, même s’il l'a juste mentionné dans l'Iliade sans s'y attarder (VI, 289-292). S'accordant par ce détail avec les prêtres, considérés dans l'Enquête comme une source fiable ${ }^{17}$, le plus grand des rhapsodes montrait

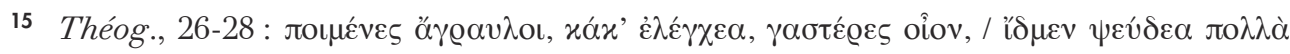

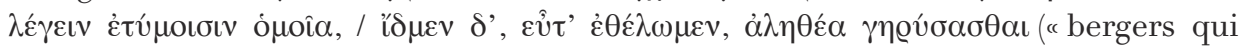
passez vos vies dans les champs, vils objets de honte, qui êtes tels des ventres, nous savons dire de nombreux mensonges qui semblent véridiques et nous savons, à chaque fois que nous le voulons, faire entendre des vérités »).

16 Sur la vérité dissimulée en dessous du mythe, voir P. Veyne, Les Grecs ont-ils cru à leurs mythes?, Paris, Seuil, 1983, p. 59.

17 L'évaluation positive qu'Hérodote donne des prêtres apparaît à différents endroits :

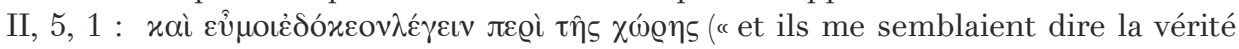




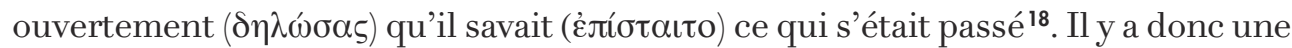
vérité historique dissimulée dans son œuvre, partiellement éclipsée par un voile de légende propre à l'épopée. Cette vérité, le “ père de l’histoire » entendait bien la faire émerger en croisant les vers homériques au témoignage de ses informateurs égyptiens. Nous nous proposons ici d'examiner, à travers différents exemples, la manière dont il tente de faire dialoguer ces deux sources.

\section{Le syncrétisme des figures mythico-historiques}

Le chapitre 112 offre un premier exemple de cette tentative : Hérodote y assimile à Hélène une divinité adorée sur les côtes d'Égypte, “ l'Aphrodite Étrangère » ${ }^{19}$. Bien entendu, on a aujourd'hui rejeté cette interprétation, reconnaissant dans cette “Aphrodite » Astarté, la déesse phénicienne de l'amour et de la guerre ${ }^{20}$.

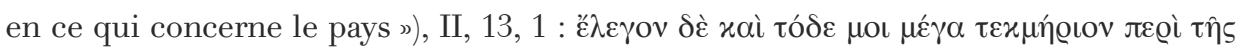

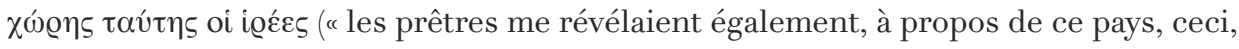

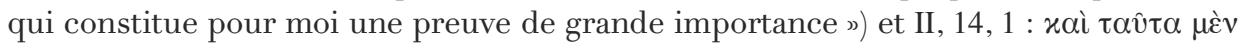

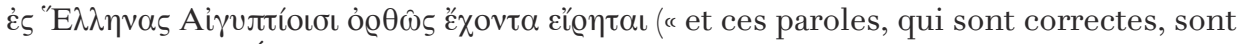
délivrées par les Égyptiens aux Grecs »).

18 Ajoutons que le père de l'histoire insiste sur cette idée, puisque ces deux lemmes se

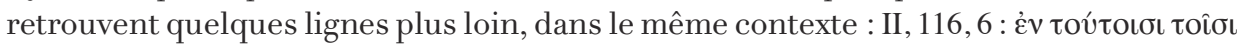

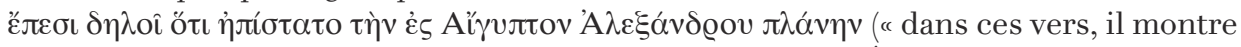
qu'il connaissait la course errante d'Alexandre [sc. Pâris] en Égypte »).

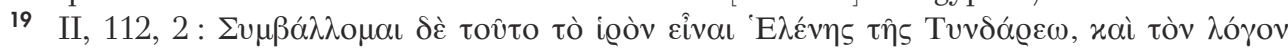

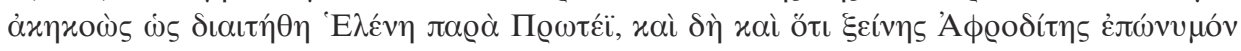

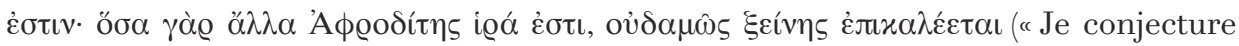
que ce sanctuaire est celui d'Hélène, fille de Tydare, après avoir également entendu le discours selon lequel Hélène aurait séjourné chez Protée, surtout parce qu’il est voué à Aphrodite Étrangère. En effet, pour ce qui est de toutes les autres choses qui sont consacrées à Aphrodite, elle n'est jamais appelée 'Étrangère' »).

20 Pour cetteidentification, voir Legrand, Hérodote, p. 139, n. 5 ; Lloyd, Commentary, p. 45 ; D. Asheri- A. B. Lloyd - A. Corcella, A Commentary on Herodotus: Books I-IV, Oxford, Oxford University Press, 2007, p. 322. Le commentaire de Lloyd esquisse un panorama complet de la question en renvoyant à toute la bibliographie qui s'y rapporte. Selon lui, “Astarté de Syrie » - 'str(t) HZrw - était une divinité sémitique honorée en Égypte à partir de la XVIII ${ }^{e}$ dynastie, où elle était la plupart du temps identifiée à Sekhmet, épouse de Ptah, mais aussi à Hator et Isis (p. 45). À laquelle de ces trois divinités les prêtres avaient-ils assimilé l'Astarté vénérée dans le sanctuaire dont parle Hérodote? Sekhmet apparaît d'emblée comme une excellente candidate, puisqu'Hérodote évoque la présence d'un “ téménos de Protée » à proximité du sanctuaire d'Hélène (II, 112, 1), une expression qui aurait pu désigner le temple de Ptah selon plusieurs chercheurs (pour une liste complète, voir Lloyd, Commentary, p. 44). Qui plus est, elle est à la fois 
Mais ce témoignage présente un grand intérêt pour comprendre la méthode de l'historien : face à des réalités étrangères, il semble se servir du mythe comme d'une grille de lecture, pour mettre en lien des figures barbares avec des dieux et héros du monde grec. Ainsi, le témoignage d'Homère, selon lequel Hélène, la plus belle des femmes, se serait rendue au pays du Nil (Od., IV, 227-228), l'aurait poussé à considérer que le sanctuaire qu'il avait vu pendant ses voyages était consacré à cette dernière. Il faut cependant noter un détail interpellant : dans l'Enquête, la reine de Sparte séjourne auprès de Protée, qui est présenté comme un pharaon et non comme une créature merveilleuse, alors qu'elle réside chez Thon dans l'Odyssée (IV, 227-228) ${ }^{21}$. Ce personnage, qu'Hérodote appelle Thonis, se trouve chez lui dans le rôle de gardien de la branche Canopique (II, 113, 3) ${ }^{22}$, alors qu'Homère ne précise pas son statut. Comment expliquer cet étonnant

une déesse guerrière, comme l'Astarté phénicienne, et une personnification de l'œil du soleil, ce qui a pu concourir à la rapprocher d'Hélène. En effet, l'historien a pu associer le nom de l'héroïne à l'idée de lumière, d'éclat, de brillance, puisque 'E $\lambda \varepsilon ́ v \eta$ ressemble au substantif $\dot{\varepsilon} \lambda \alpha ́ v \eta$, “ le flambeau » (qui peut d'ailleurs parfois s'écrire $\dot{\varepsilon} \lambda \varepsilon ́ v \eta$ ) ainsi qu'à

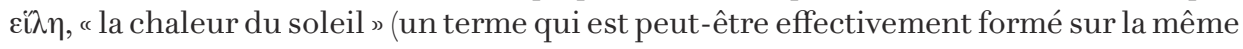
racine que le prénom “ Hélène », en l'occurrence une racine *swel qui correspondrait au sanskrit $s v a r-a-t i$, «il allume »). Si de tels rapprochements sont aujourd’hui considérés comme douteux, ils ont pu être établis par un Ancien comme Hérodote qui ne disposait pas des mêmes acquis que les Modernes en termes d'étymologie (pour une synthèse du débat concernant la signification du prénom " Hélène », voir R. BEEkes, Etymological Dictionary of Greek, Leyde - Boston, Brill, coll. « Leiden Indo-European Etymological Dictionary Series », 2010, p. 406-407). Considérer que ce chapitre de l'Enquête fait référence à Sekhmet est donc tentant, mais il pourrait tout aussi bien s'agir d'Hator, déesse de l'amour et de la beauté, ce qui expliquerait que l'on ait présenté à l'historien le lieu comme consacré à “ Aphrodite ». Lui-même aurait assimilé la divinité à la plus belle des héroïnes du monde grec. Ce deuxième schéma semble lui aussi satisfaisant. On voit mal, en revanche, comment une déesse funéraire telle qu'Isis pouvait être associée à une déesse de l'amour et de la guerre comme Astarté. Le phénomène viendrait, selon nous, d'une confusion des Égyptiens eux-mêmes entre Isis et Hator, qui portaient toutes les deux sur la tête un disque solaire inséré entre deux cornes de vache.

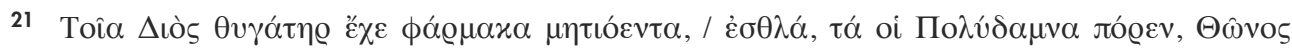

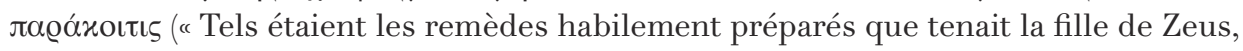
ces bons remèdes que lui procura Polydamna, l'épouse de Thon »).

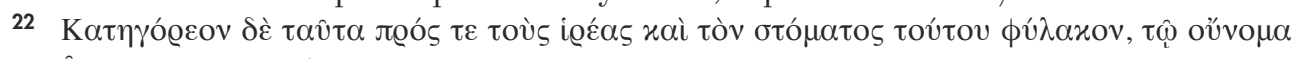

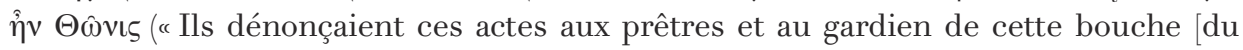
fleuve], dont le nom était Thonis »). Sur la question de savoir à quelle fonction officielle de la société égyptienne Hérodote fait allusion en parlant de ce " gardien », voir LLOYD, Commentary, p. 49 ; Asheri et al., Commentary, p. 323. 
phénomène? Legrand, dans son édition, suggère une réponse convaincante à cette question :

“ Peut-être la présence dans le téménos d'une statue de pharaon représenté sous les traits du dieu Nil (cf. Spiegelberg, Mélanges Loret, p. 103-106) ou la représentation - dans un sanctuaire du quartier des Tyriens - du dieu-poisson Dagon, concoururent-elles à créer le personnage du roi d'Égypte Protée et à établir une relation entre lui et le temple memphite. „²3

Ainsi, les représentations iconographiques qu'Hérodote avait vues durant son voyage auraient été à l'origine de cette inversion des rôles. Des deux possibilités proposées par Legrand, nous serions enclin à préférer celle de l'amalgame de Protée avec le pharaon/Nil, nous représentant la scène comme ceci. Arrivé dans le sanctuaire de Memphis, l'historien a pu être frappé par une étrange statue et aurait alors demandé à ses informateurs de qui il s'agissait. Entendant que c'était l'un de leurs anciens rois et le voyant associé à une composante liquide, Hérodote aurait aussitôt assimilé ce dernier à la figure de Protée, qualifié de « vieillard de la mer » dans l'Odyssée (IV, 365 et 384) ${ }^{24}$. Pensant qu'une vérité se dissimulait sous le voile mensonger du mythe, il a pu croire qu'Homère avait transformé en être merveilleux un monarque que ses sujets vénéraient comme un dieu et auquel était attribué le pouvoir de contrôler les eaux du Nil. Dès lors, Thon aurait pu apparaître comme un haut fonctionnaire qui jouissait d'un certain prestige auprès du pharaon. Le gardien de la branche Canopique devait fournir à Hérodote un candidat tout trouvé pour ce rôle. La reconstitution de ce scénario, qui donnerait raison à Spiegelberg, semble être un moyen assez habile d'expliquer la situation, d'autant que cela cadrerait avec la méthode que l'on reconnaît communément à l'historien. C'est, en effet, un fait établi que l'auteur de l'Enquête accorde plus de crédit à l'ő $\psi \varsigma \varsigma$ (ce qu'il voyait de ses propres yeux) qu'à l'ảxoń (les discours qu'il entendait de ses informateurs) ${ }^{25}$.

23 Legrand, Hérodote, p. 139, n. 2. Certains chercheurs ont également pensé que l'amalgame serait dû à un titre royal, p̧rwty, qu'Hérodote aurait rapproché de “Protée » en raison des ressemblances phonétiques (pour une liste des partisans de cette hypothèse, voir LLoyd, Commentary, p. 43). Lloyd signale toutefois que rien ne prouve que ce terme pouvait être employé dans ce sens, signifiant originellement « les deux portes ». Il est tentant de croire qu'un tel mot aurait d'abord pu désigner le palais royal pour ensuite s'appliquer au monarque qui y habitait, de même que pour " pharaon » qui signifiait à l'origine “ grande maison ». Ce n'est pourtant qu'une théorie incertaine.

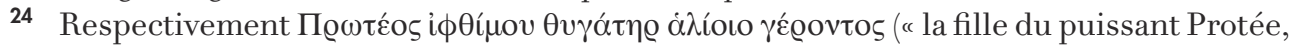

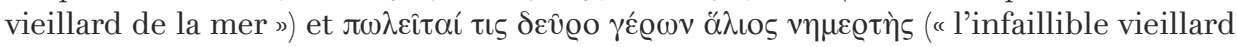
de la mer va et vient en ce lieu »).

25 Selon G. Schepens, $L$ '“ autopsie » dans la méthode des historiens grecs du ve siècle avant 


\section{Le sacrifice de Ménélas}

Ces syncrétismes semblent témoigner d'une véritable volonté de faire concorder les propos de ses informateurs avec le récit d'Homère. Cette démarche expliquerait également pourquoi Hérodote rapporte sans la critiquer l'histoire relative au passage des héros de la guerre de Troie en Égypte, qu'un lecteur moderne aurait peine à croire. Celle-ci présente en effet un côté manichéen assez visible : Protée y est présenté comme un roi à la fois juste et clément, véritable modèle de droiture qui contraste avec Pâris et Ménélas. Après avoir convaincu le prince troyen de son crime et lui avoir pris Hélène ainsi que les trésors volés à Sparte, le pharaon l'aurait laissé repartir en Asie, sans lui infliger un châtiment digne de ses méfaits (II, 115, 4-6). Ensuite, le monarque égyptien aurait rendu à Ménélas ses biens et son épouse, qu'il avait bien traitée (II, 119, 1). L'Atride, en revanche, aurait répondu à ce bienfait par un acte cruel : comme il lui était impossible de naviguer, il aurait immolé deux enfants du pays pour pouvoir reprendre la mer (II, 119, 3). Un tel récit semble témoigner de l'orgueil national égyptien, puisque les prêtres opposent au roi vertueux de leur pays les étrangers présentés sous un jour clairement défavorable, qu'il s'agisse du Troyen Alexandre (Pâris) ou du Grec Ménélas.

Il est donc étonnant qu'Hérodote ne conteste pas ce scénario, ce qui incite à tenter de comprendre cette absence de condamnation. Pour Plutarque, la solution est simple : l'historien aurait été favorable aux barbares ( $\phi \iota \lambda \circ \beta \alpha ́ \varrho \beta \alpha \varrho o \varsigma)$ au point de ne pas contredire leurs témoignages, même les plus absurdes ${ }^{26}$.

J.-C., Bruxelles, Palais des Académies, 1980), Hérodote aurait été le premier à percevoir “ l'importance historico-méthodologique de l'autopsie » (p. 196). J. BoËLdiEu-TrÉvet et D. Gondicas, Lire Hérodote, Rosny-sous-Bois, Éditions Bréal, 2005 résument, quant à elles, cette pratique comme suit : ‘ La vue prime sur l'écoute et l'auteur s'efforce de n'accorder foi à l'information recueillie directement auprès d'informateurs [...] que dans la mesure où il peut en contrôler l'exactitude par la vue. » (p. 24) Plutarque, De Herodoti malignitate, 857b. Personne ne contestera que cette position semble extrême. Cependant, elle pourrait fournir un début d'explication au phénomène qui nous occupe : la présentation favorable du pharaon pourrait être en partie due à l'admiration qu'Hérodote vouait aux Égyptiens. Il faut en effet rappeler que l'émerveillement face aux peuples habitant les extrémités de la terre est reconnu comme général dans le monde grec, cf. J. S. Romm, The Edges of the Earth in Ancient Thought, Princeton, Princeton University Press, 1992 ; D. Fehling, "The Art of Herodotus and the Margins of the World ", in Z. R. W. M. von Martels (éd.), Travel Fact and Travel Fiction: Studies on Fiction, Literary Tradition, Scholarly Discovery, and Observation in Travel Writing, Leyde, Brill, 1994, p. 1-15; H.-G. Nesselrath, " Herodot und die Enden der Erde ", $M H 52$ (1995), p. 20-44. Cette attitude aurait pu pousser 
Nous aurions plutôt tendance à penser que le discours des prêtres lui semblait vraisemblable et qu'il ne voyait donc aucune raison de s'y opposer. Il faut en effet rappeler que le domaine du " possible » varie fortement d'une époque à l'autre et que le "merveilleux » a pleinement sa place dans l'Enquête, comme l'a bien montré Munson ${ }^{27}$. Dans le cas qui nous occupe, ce sacrifice aurait pu apparaître comme plausible à Hérodote, étant donné que les faits étaient censés avoir eu lieu au temps des héros de la guerre de Troie, une époque où Agamemnon luimême avait immolé sa propre fille à Aulis. Rappelons que, contrairement aux modernes, l'écrivain d'Halicarnasse ne devait pas trouver incroyable le meurtre de victimes humaines, puisque certaines peuplades mentionnées dans l'Enquête conservaient de telles pratiques à son époque, comme les Scythes (IV, 62). Qui plus est, le témoignage des prêtres n'entre pas en contradiction avec celui d'Homère, étant donné que ce dernier ne précise pas la nature des victimes de Ménélas, se contentant de dire que l'Achéen avait longtemps attendu sur les rivages d'Égypte à cause de vents contraires $(O d .$, IV, 351-352) et que son départ ne fut possible qu'après l'accomplissement d'un sacrifice (Od., IV, $472-473$ et 478-479). Hérodote n'avait donc aucune raison de douter des propos de ses informateurs, qui n'avaient rien d'invraisemblable pour lui et ne s'opposaient pas au récit homérique. Au contraire, ils complétaient l'histoire du rhapsode, qui en connaissait peut-être plus qu'il ne le montrait mais choisissait en âme et conscience de laisser de côté certains éléments. Si tel était bien le regard que l'historien portait sur Homère, on peut comprendre qu'il n'ait vu aucun souci à agréger à son témoignage de nouvelles données collectées dans les contrées étrangères.

\section{La cause de la guerre de Troie}

Cependant, il semble à priori que cette tentative de conciliation des sources soit absente du passage où Hérodote fait part de son avis sur la guerre de Troie (II, 120). Il y donne en effet raison aux Égyptiens, selon lesquels Hélène ne se serait jamais trouvée dans la ville de Priam, qui aurait donc été détruite sur un malentendu. Les Grecs auraient cru que les Troyens se moquaient d'eux en prétendant que la reine de Sparte était restée chez Protée et auraient assiégé la cité jusqu'à la victoire où ils auraient constaté que cela était vrai (II, 118). Une telle version contraste avec celle d'Homère où Hélène se trouvait bien à Ilion, ce qui donne envie de croire que l'historien n'accorde ici aucun crédit au rhapsode. Pourtant, on peut noter

l'historien à reprendre le récit des prêtres, voire à en intensifier la composante manichéenne.

27 R. V. Munson, Telling Wonders: Ethnographic and Political Discourses in the Work of Herodotus, Ann Arbor, University of Michigan Press, 2001. 
que ce qu'il se propose d'ajouter au témoignage des prêtres se fonde sur différents détails de l'Iliade.

Tout d'abord, comme l'a remarqué Legrand ${ }^{28}$, les propos selon lesquels les Troyens auraient rendu Hélène aux Achéens si elle s'était trouvée dans leur ville, et ce même contre le gré de Pâris (II, 120, 1$)^{29}$, font écho aux paroles prononcées par les vieillards sur les remparts de la ville (Il., III, 159-160) ${ }^{30}$. Nous pourrions ajouter à ce rapprochement qu'il est dans les deux cas question des citoyens et de leurs enfants qui semblent menacés par un futur désastre (II, 120, 2) (11 $^{31}$ au

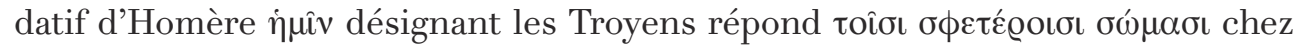

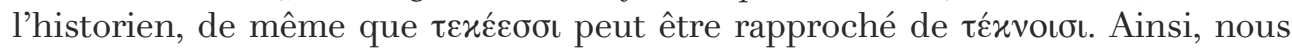
nous trouvons en présence de deux mots au même cas et dans le même ordre pour exprimer la même idée, ce qui pourrait constituer un écho supplémentaire à l'œuvre d'Homère, bien qu'il puisse s'agir d'une simple coïncidence.

L'idée selon laquelle Hector, en digne héritier du pouvoir royal, ne pouvait pas décemment laisser impunis les méfaits de son frère et permettre que tout son peuple coure un grand danger (II, 120, 4) ${ }^{32}$ tire peut-être aussi sa source de l'Iliade. Dans cette œuvre, Hector, après avoir criblé d'insultes son frère (III, 39-40), lui reproche les torts causés à son peuple (III, 50-51) (33 $^{33}$ lui feraient mériter d'être puni de lapidation (III, 56-57). Notons qu'à l'expression xaì toî

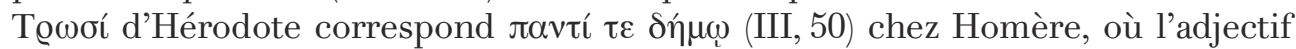
$\pi \tilde{a} \varsigma$ se trouve au même cas pour désigner les mêmes personnes. Ajoutons à cela les vers affirmant que les Troyens avaient Pâris en horreur (III, 453-454), encore un élément qui aurait pu amener Hérodote à croire que la seule cause possible de la

28 Legrand, Hérodote, p. 145, n. 2.

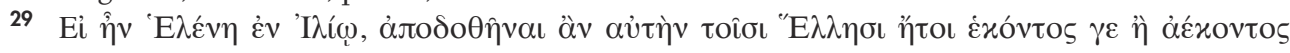

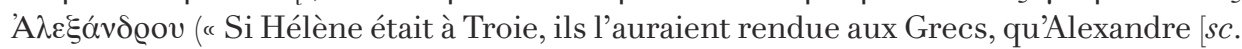
Pâris] l'ait voulu ou non »).

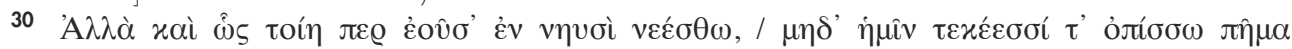
$\lambda i ́$ roı nous laisse pas de souffrance à nous et à nos enfants par la suite »).

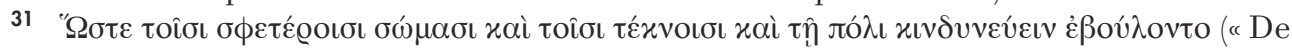
façon à ce qu'ils aient voulu faire courir un danger à leur propre personne, à leurs enfants et à leur ville »).

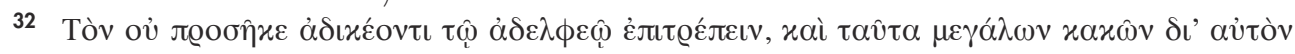

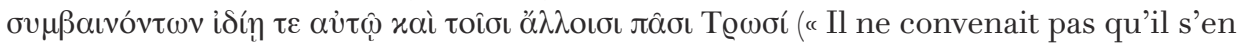
remette à son frère qui s'était montré injuste, et ce alors qu'à cause de ce dernier, de grands maux arrivaient à Hector lui-même et à tous les autres Troyens »).

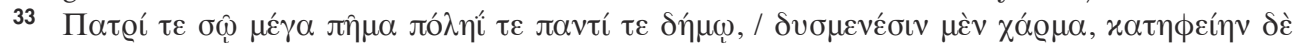

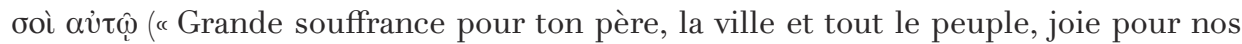
ennemis, honte pour toi-même »). 
guerre de Troie serait la suivante : les Achéens n'auraient pas cru les Dardanides qui leur affirmaient qu'Hélène n'était pas auprès d'eux (II, 120, 5).

Cette méprise qui aurait été à l'origine de la guerre serait, selon l'historien, due à la volonté des dieux, un avis qu'il présente comme “personnel » (II, $120,5)^{34}$. Pourtant, cette idée du sort imposé par les puissances divines, malgré les tentatives des hommes pour y échapper, apparaît comme un véritable lieu commun qui trouve, entre autres, de nombreux échos dans l'œuvre homérique. Citons seulement ici le prologue de l'Tliade, où les événements racontés sont

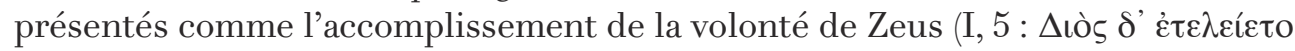

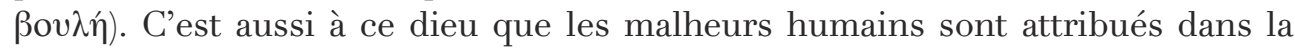
section de l'Odyssée consacrée au dîner de Télémaque chez Ménélas (IV, 236-237).

Et ce ne sont pas là les seules références aux dieux bien décidés à voir la guerre se poursuivre malgré tous les efforts des Achéens et des Troyens pour trouver un terrain d'entente, comme le montrent les nombreux exemples des chants III et IV de l'Iliade. Ainsi, Priam dit à Hélène qu'elle n'est pas responsable de la tournure des événements, mais que cela relève des dieux (III, 164). Plus loin, il est affirmé explicitement que Zeus ne souhaite pas voir le désaccord se résoudre par un combat singulier, alors que les deux camps en présence semblent favorables à cette option (III, 302). Il n'est par ailleurs pas le seul, puisqu'Aphrodite, craignant de voir périr son cher Pâris, le soustrait à la violence de Ménélas (III, 380-382), tandis que sa mort aurait pu mettre un terme à la situation conflictuelle. De même, Héra intervient pour pousser son époux à faire éclater la guerre (IV, 64-67). C'est alors qu'Athéna entre elle aussi en scène, une fois que le Cronide lui a confié la mission de pousser les Troyens à violer le traité (IV, 70-72), en manipulant Pandare dont le trait déclenche les hostilités (IV, 93-103). Ajoutons enfin que, dans ce chant, Zeus est désigné comme l'arbitre des combats entre humains (IV, 84). Tous ces éléments amènent donc à penser que l'opinion “ personnelle » d'Hérodote sur les origines de la guerre de Troie serait en vérité influencée par la conception traditionnelle selon laquelle les dieux - et surtout Zeus - fixeraient le malheur des hommes. Certes, cette idée était commune aux Grecs, mais les échos aux deux épopées homériques que nous avons mis en évidence incitent à croire que l'historien s'est ici encore inspiré du plus grand des rhapsodes.

Cette notion vague d' “ inspiration » mérite d'ailleurs d'être précisée, puisqu'elle peut recouvrir des réalités diverses. Dans ce chapitre 120, nous observons en effet deux manières différentes dont Hérodote “s'inspire " d'Homère. Il peut s'agir, d'une part, de la reprise d'une idée, comme celle dont il vient d'être question: lorsque l'auteur de l'Enquête fait de la divinité la responsable de la guerre de Troie,

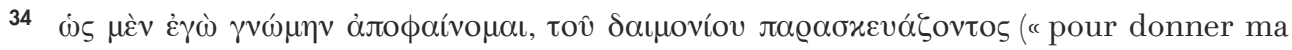
propre opinion, cela a été causé par une divinité »). 
il reprend simplement à son compte la conception exprimée à de nombreuses reprises par l'aède, sans la remettre en question. À côté de cela, l'argumentaire déployé par l'historien pour prouver qu'Hélène ne s'est jamais rendue à Ilion indique un tout autre rapport avec l'œuvre homérique. La présence de la reine de Sparte dans la ville est clairement remise en cause sur la base de différents éléments qui apparaissent également dans l'Tliade: les paroles des vieillards et d'Hector ainsi que la mention de la haine des Troyens pour Pâris. Nous pourrions donc dire, de façon assez plaisante, qu'Hérodote tente de “ combattre Homère par Homère », en l'occurrence de critiquer un point important de son récit en s'appuyant sur d'autres points moins importants.

\title{
Conclusion
}

Les trois exemples présentés dans cet article illustrent les rapports que le “ père de l'histoire » entretenait avec le mythe. Nous sommes en droit de nous demander si sa façon de lire et de comprendre Homère ne s'inscrit pas dans le cadre d'une attitude générale face à l'héritage épique qui avait cours au v siècle. Une telle hypothèse semble tentante compte tenu du contexte intellectuel dans lequel évoluait l'historien. Devant le relativisme d'un sophiste comme Protagoras, qui mettait en exergue la diversité des opinions, Hérodote a pu constater que la même diversité apparaissait dans la tradition mythique, où plusieurs versions d'un même récit coexistaient. Il ne s'est cependant pas limité à ce constat, tentant de confronter les différents témoignages pour distinguer ce qui semblait plausible et ce qui relevait du fantasme. Cette attitude rappelle celle de Socrate qui, contrairement aux sophistes, croyait qu'une vérité destinée à rester une et immuable se cachait derrière des discours multiples et divergents. Cela donne à penser que le philosophe n'était pas le seul à envisager la recherche de la connaissance de cette manière. Mais tout cela reste hypothétique et mériterait des investigations beaucoup plus poussées. Nous nous limiterons à dire, au terme de cet article, qu'Hérodote accorde apparemment une certaine légitimité à Homère devant la multiplicité des points de vue. Il semble essayer de le récupérer en le réinterprétant lorsque ses propos semblent peu crédibles. Même si l'historien le critique, il ne le considère pas moins, à sa manière, comme un « maître de vérité ».

\author{
Arnaud Amilien \\ 65 rue de la Gade \\ 7141 Carnières (Belgique) \\ arnaudfefletef@hotmail.com
}




\section{Bibliographie}

D. Asheri - A. B. Lloyd - A. Corcella, A Commentary on Herodotus: Books I-IV, Oxford, Oxford University Press, 2007.

A. Ballabriga, “ La question homérique : pour une réouverture du débat », $R E G$ 103 (1990), p. 16-29.

R. BeEkes, Etymological Dictionary of Greek, Leyde - Boston, Brill, coll. « Leiden Indo-European Etymological Dictionary Series », 2010.

D. Boedecker, “ Herodotus' Genre(s) », in M. Depew - D. Obbink (éds), Matrices of Genre. Authors, Canons, and Society, Cambridge (MA), Harvard University Press, 2000, p. 97-114.

D. Boedecker, "Epic Heritage and Mythical Patterns in Herodotus », in E. J. Bakker - I. J. F. de Jong - H. van Wees (éds), Brill's Companion to Herodotus, Leyde - Boston - Cologne, Brill, 2002, p. 97-116.

J. Boëldieu-Trévet - D. Gondicas, Lire Hérodote, Rosny-sous-Bois, Éditions Bréal, 2005.

C. Brillante, « Myth and History: History and the Historical Interpretation of Myth », in L. Edmunds (éd.), Approaches to Greek Myth, Baltimore, JHU Press, 1990, p. 91-140.

P. Cartledge - E. Greenwood, “ Herodotus as a Critic: Truth, Fiction, Polarity », in E. J. Bakker - I. J. F. de Jong - H. van Wees (éds), Brill's Companion to Herodotus, Leyde - Boston - Cologne, Brill, 2002, p. 351-386.

L. Couloubaritsis, Aux origines de la pensée européenne, de la pensée archaïque au néoplatonisme, Bruxelles, De Boeck-Wesmael, $2003^{4}$.

M. Detienne, Les maîtres de vérité dans la Grèce archaïque (préface de Pierre Vidal-Naquet), Paris, Maspero, 1967.

J.-F. Dortier, “ Y a-t-il eu un miracle grec ? », in T. Lepeltier (éd.), Histoire et philosophie des sciences, Auxerre Cedex, Sciences Humaines Éditions, 2011, p. 11-20.

D. Fehling, Herodotus and his « Sources »: Citation, Invention, and Narrative Art, Leeds, Cairns, 1989 (orig. all. : Die Quellenangaben bei Herodot, 1971).

D. Fehling, " The Art of Herodotus and the Margins of the World », in Z. R. W. M. von Martels (éd.), Travel Fact and Travel Fiction: Studies on Fiction, Literary Tradition, Scholarly Discovery, and Observation in Travel Writing, Leyde, Brill, 1994, p. 1-15.

L.Ferreri, La questione omerica dal Cinquecento al Settecento, Roma, Edizioni di storia e letteratura, 2007.

M. I. Finley, “ Myth, Memory, and History », in M. I. Finley (éd.), The Use and Abuse of History, New York, The Viking Press, 1975, p. 1-33.

R. Fowler, "The Homeric Question », in R. Fowler (éd.), The Cambridge Companion to Homer, Cambridge, Cambridge University Press, 2004, p. 220-232. 
M. Hauskeller, Mythologies of Transhumanism, Bâle, Springer International Publishing AG, 2016.

D. Lateiner, The Historical Method ofHerodotus, Toronto, University of Toronto Press, 1989.

Ph.-E. Legrand, Hérodote. Histoires, II, Paris, Les Belles Lettres, CUF, 1932.

A. B. Lloyd, Herodotus. Book II, Commentary 99-182, Leyde - New York Copenhague - Cologne, Brill, 1988.

J. Marincola, Authority and Tradition in Ancient Historiography, Cambridge, Cambridge University Press, 1997.

R. V. Munson, Telling Wonders: Ethnographic and Political Discourses in the Work of Herodotus, Ann Arbor, University of Michigan Press, 2001.

G. NAGy, Homeric Questions, Austin, University of Texas Press, 1996.

H.-G. Nesselrath, « Herodot und die Enden der Erde », MH 52 (1995), p. 20-44.

W. Nestle, Vom Mythos zum Logos, Stuttgart, Kröner, 1940.

J. S. Romm, The Edges of the Earth in Ancient Thought, Princeton, Princeton University Press, 1992.

S. SAÏD - M. TRÉDÉ- A. Le Boulluec, Histoire de la littérature grecque, Paris, PUF, coll. " Quadrige manuels », 1997.

G. Schepens, L'“ autopsie » dans la méthode des historiens grecs du Ve siècle avant J.-C., Bruxelles, Palais des Académies, 1980.

R. Thomas, "Performance and Written Publication in Herodotus and the Sophistic Generation ", in W. Kullmann - J. Althoff (éds), Vermittlung und Tradierung von Wissen in der griechischen Kultur, Script-Oralia 61, Tubingue, 1993, p. 225-244.

H. Verdin, « Les remarques critiques d'Hérodote et Thucydide sur la poésie en tant que source historique ",Historiographia antiqua: commentationesLovanienses in honorem W. Peremans septuagenarii editae, Leuven, Leuven University Press, coll. "Symbolae Facultatis Litterarum et Philosophiae Lovaniensis », 1977, p. 53-76.

J.-P. Vernant, Les Origines de la pensée grecque, Paris, PUF, coll. « Mythes et Religions ", 1962.

P. Veyne, Les Grecs ont-ils cru à leurs mythes ?, Paris, Seuil, 1983.

K. H. Waters, Herodotus the Historian: His Problems, Methods and Originality, Londres - Sydney, Routledge, 1985. 

ANABASES

Traditions et Réceptions de l'Antiquité

Revue de l'équipe de recherche E.R.A.S.M.E.

Université Toulouse-Jean Jaurès (UT2J)

NoRMES RÉDACTIONNELLES

ANABASES publie des articles dans cinq langues : français, anglais, allemand, italien et espagnol.

Les articles ne dépasseront pas 35000 signes et seront conformes aux normes de la revue,

disponibles sur le site web : http://plh.univ-tlse2.fr

Les articles seront pourvus d'un résumé en français et en anglais, ainsi que de six à huit mots-clés dans ces deux langues.

Les articles pourront être accompagnés de planches en noir et blanc.

Les comptes rendus compteront de 4500 à 6000 signes.

Site web avec Présentation, Sommaire de tous les Numéros et Bulletin d’Abonnement

http://plh.univ-tlse2.fr

Revues.org : http://anabases.revues.org

\section{Courrier}

Pour les articles :

Clément Bur (clement.bur@univ-jfc.fr)

Catherine VALENTI (catherine.valenti@univ-tlse2.fr)

Pour les comptes rendus :

NoémieVILlacÈQue (noemie.villaceque@univ-reims.fr)

Université Toulouse-Jean Jaurès (UT2J)

Équipe P.L.H. - E.R.A.S.M.E. (EA4601)

Maison de la recherche (MdR)

5, allées Antonio Machado

F-31058 Toulouse Cedex 9

Tél. : 0033/(0)5.61.50.25.56 et 57

Fax : 0033/(0)5.61.50.24.90 


\section{Historiographie et identités culturelles}

Carole QuATRELIVRe, Le sanctuaire gaulois de Gournay-sur-Aronde (Oise). Retour sur une découverte exceptionnelle des années 1970

\section{Traditions du patrimoine antique}

Christophe CorbIER, Le pindarisme et l'archéologie musicale : style, valeur et authenticité de la première Pythique à l'époque moderne

Arnaud AmiLien, Hélène en Égypte : Hérodote en dialogue avec l'épopée

\section{Archéologie des savoirs}

Sébastien CAzalas, Au jardin des exempla. Rhétorique et stratégie de l'exemplum antique dans l'œuvre politique de Jean Juvénal des Ursins (1388-1473)

Dossier - Perpétuer Ovide : aspects moraux, éditoriaux, linguistiques et culturels $\left(\mathrm{xIV}^{\mathrm{e}}-\mathrm{XvIII}{ }^{\mathrm{e}} \mathrm{s}\right.$.)

Francesca DelL'Oro, Introduction

Hélène Casanova-Robin, L'audace châtiée : Phaéton, Actéon et Icare dans la tradition latine jusqu'à la Renaissance, tours et détours d'un symbolisme

Dylan Bovet, Le commentaire latin des Métamorphoses d'Ovide : pratiques humanistes et évolutions de Regius-Micyllus (1543) à Burmann-Heinsius (I727)

Martine Furno, Ovide en classe, ou un auteur en éclats

Basil Neurs, D'un Ovide chrétien à un Ovide burlesque, du Moyen Âge au Grand Siècle : continuités et changements dans la traduction et dans l'illustration des Métamorphoses perçus à travers deux éditions du xvir siècle

Olivier ThÉvenaz, Épilogue

\section{Actualités et débats}

Tiphaine-Annabelle BESNARD, 2019 : l'année pop des musées d'archéologie. Retour sur l'exposition romaine Il classico si fa pop. Di scavi, copie e altri pasticci

\section{Relire les classiques des sciences de l'Antiquité}

Jean-Pierre AıBERT, Le premier Detienne : une relecture de « La notion mythique d'A $\lambda \hat{\eta} \theta \varepsilon\llcorner\alpha$ » (REG, 1960, p. 27-35)

Marcel DetIENNE, La notion mythique d'A $\lambda \hat{\eta} \theta \varepsilon \varepsilon \alpha$

\section{L'atelier de l'histoire : chantiers historiographiques}

Antiquités numériques (coordonné par Élodie Guillon) (1)

Élodie GuILLON, Introduction

Jaime ALvar, Le projet EPIDI : Epítetos divinos. Experiencia religiosay relaciones de poder en Hispania

Les mots de l'Antiquité (coordonné par Magali Soulatges) (10)

Jack Tномаs, L’Antiquité dans les toponymes de l’État de New York

Actualité du théâtre (coordonné par Malika Bastin-Hammou) (4)

Mathieu Ferrand, “Avons-nous perdu le Soleil ? / Ou l'avons-nous chassé ? ”

Thyeste de Sénèque, traduit par Florence Dupont. Mise en scène de

Thomas Jolly (Avignon, 2018).

Voyages et Voyageurs (coordonné parVéronique Krings) (11)

Claude Azıza, Freud à Pompéi

\section{Comptes rendus de lecture}

